\title{
Tumor budding as a standardized parameter in gastrointestinal carcinomas: more than just the colon
}

\author{
Kyra B Berg ${ }^{1} \cdot$ David F Schaeffer ${ }^{1,2}$
}

Received: 7 September 2017 / Revised: 4 January 2018 / Accepted: 7 January 2018 / Published online: 6 February 2018

(c) United States \& Canadian Academy of Pathology 2018

\begin{abstract}
Tumor budding, defined as single cells or clusters of less than five cells, is thought to be a histomorphologic marker of an aggressive tumor behavior mimicking the embryologic epithelial-mesenchymal transition, and has been well established in the past two decades as a poor prognostic factor in colorectal carcinoma. Slow uptake in routine reporting of this important pathologic prognostic feature was in part due to differing methods of assessment of budding reported in the literature, but has recently been clarified at a consensus conference on tumor budding in colorectal carcinoma. Tumor budding is also increasingly being reported as a useful pathologic prognostic feature in other gastrointestinal carcinomas, including esophageal squamous cell carcinoma and adenocarcinoma, gastric intestinal-type adenocarcinoma, pancreatic ductal adenocarcinoma, and ampullary adenocarcinoma. In this review, we will summarize the studies on tumor budding in gastrointestinal carcinomas, with a focus on the methods of assessment used and the potential clinical applications of the findings.
\end{abstract}

In a time of ever increasing molecular scrutiny of cancers, histomorphologic analysis remains an important tool to stratify malignant lesions. Tumor budding, defined by single cells or small clusters of cells, has been well established in the past two decades as a poor prognostic factor for colorectal adenocarcinoma, but has been slow to be incorporated into routine pathology reporting. However, the most recent AJCC 8th edition, as well as the College of American Pathologists guidelines for colorectal carcinoma reporting, are now including tumor budding as an optional reporting field with a recommendation to report in all stage I and stage II cases, in part following an international consensus conference, which provided us with a standardized reporting tool for tumor budding in colorectal carcinoma [1]. Tumor budding, however, is not unique to colorectal carcinoma and more recently has been gaining attention as a prognostic factor in a number of other gastrointestinal

Kyra B Berg

kyraberg@alumni.ubc.ca

1 Department of Pathology and Laboratory Medicine, The University of British Columbia, Vancouver, BC, Canada

2 Division of Anatomical Pathology, Vancouver General Hospital, Vancouver, BC, Canada malignancies. Here, we will review the significance of tumor budding as a prognostic factor, with a focus on the methods of assessment used and the potential clinical applicability of the results, in gastrointestinal carcinomas, including the esophagus, stomach, pancreas, ampulla of Vater, and colon.

\section{Toward a standardized assessment of tumor budding}

The first description of what we now call tumor budding was by Imai in the 1950s, as "sprouting" at the invasive edge of carcinomas [2]. Since then, tumor budding has been shown to be a histopathologic marker of an aggressive tumor biologic process. Tumor buds in colorectal carcinoma show downregulation of adhesion protein E-cadherin, accompanying nuclear translocation of beta-catenin, and loss of cell polarity with acquisition of a fibroblastoid morphology [3]. One prevalent hypothesis has been that tumor budding mimics the epithelial-mesenchymal transition, a normal process involved in embryologic development and wound healing, whereby cells reduce intercellular adhesion proteins and acquire a mesenchymal phenotype capable of migration [3, 4]. Colorectal carcinoma tumor buds have been shown to have upregulation of 


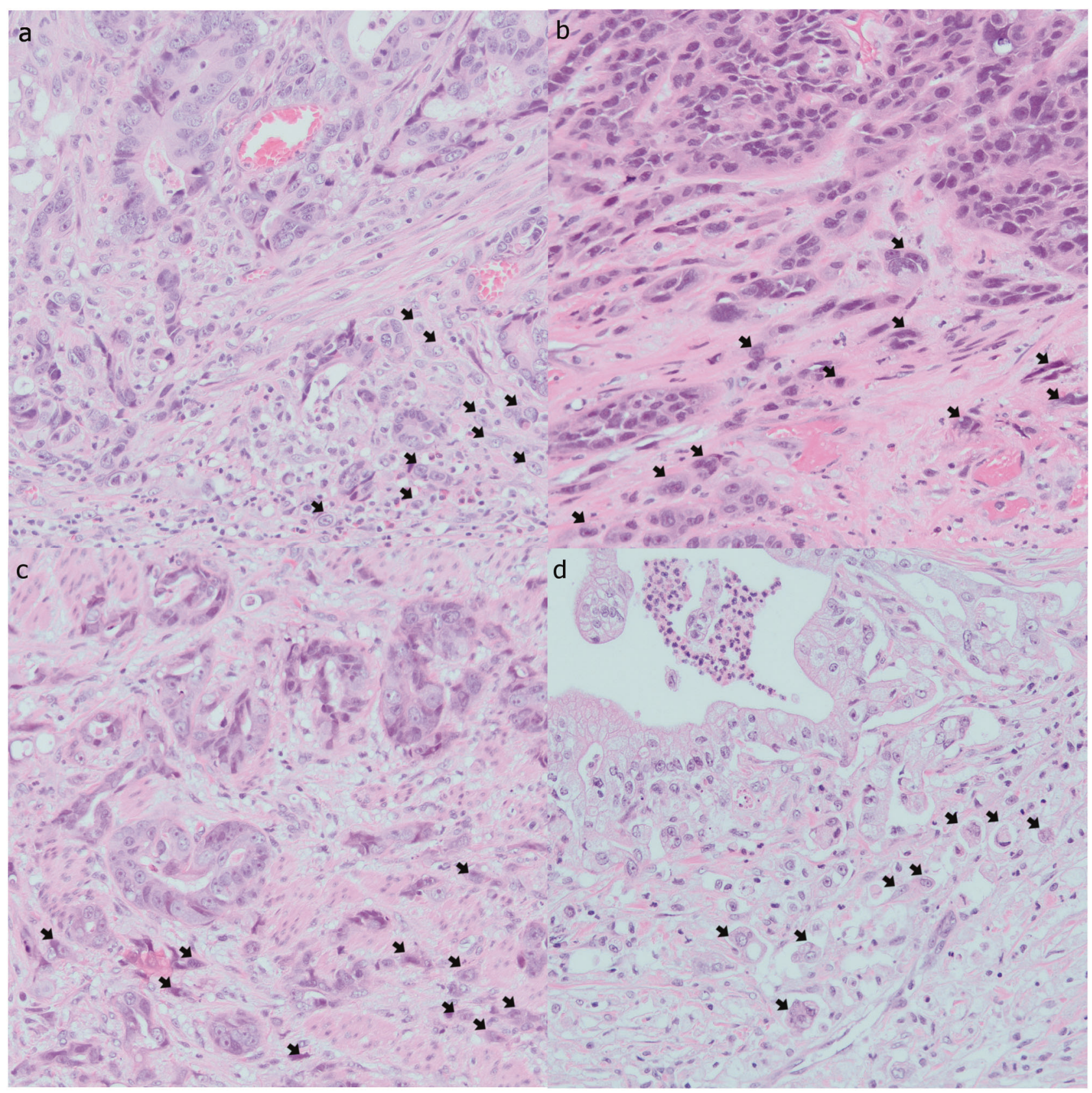

Fig. 1 Tumor budding in gastrointestinal carcinomas, with arrows to indicate budding foci. a Colorectal carcinoma (hematoxylin and eosin, $\times 200$ ). b Esophageal squamous cell carcinoma (hematoxylin and

mesenchymal markers, and of known inducers of the epithelial-mesenchymal transition such as transcription factors ZEB1 and ZEB2 [4]. Another hypothesis suggests that although tumor buds exhibit E-cadherin downregulation, they do not share other regulatory changes common to the epithelial-mesenchymal transition and that the budding phenotype may occur by another mechanism $[5,6]$.

A study by Hase et al. in 1993 first adopted the phrase "tumor budding", and showed a dramatic decrease in eosin, $\times 200$ ). c Gastric intestinal-type adenocarcinoma (hematoxylin and eosin, $\times 200$ ). d Pancreatic ductal adenocarcinoma (hematoxylin and eosin, $\times 200$ ).

survival with increased budding in colorectal carcinoma (5year survival of 22 vs. $71 \%, p<0.001$ ) [7]. This first definition of budding by Hase was subjective, with budding defined as small clusters of cells at the invasive front, and budding classified into none or mild (BD-1) and moderate or severe (BD-2) (Fig. 1).

Over the past two decades, numerous methods of assessing tumor budding have been proposed, with variations in the area of assessment, cutoff values, and use of standard hematoxylin and eosin stained slides vs. 
cytokeratin immunohistochemistry. One of the first quantitative, and more popular, methods was proposed by Ueno et al. [8] in a study looking at pathologic prognostic features of pT1 colorectal carcinoma. Their group defined tumor buds as single cells or clusters of $<5$ cells, counted buds in the area of maximal budding using a $\times 250\left(0.385 \mathrm{~mm}^{2}\right)$ field, and then classified patients into negative budding ( $<10$ buds) and positive budding ( $\geq 10$ buds). A subsequent study by Ueno et al. [9] used a modified method, counting budding in a $\times 200\left(0.785 \mathrm{~mm}^{2}\right)$ field, with five buds as the cutoff for high-grade budding. Another popular method of budding assessment was outlined by Karamitopolou et al. [10] in their study on pancreatic ductal adenocarcinoma, using a cytokeratin stain to highlight budding and averaging bud counts over 10 high power fields (hpf). One multicenter study has looked at reproducibility of tumor budding assessments on hematoxylin and eosin vs. cytokeratin staining, using the Ueno and Hase methods in addition to two other methods $[11,12]$, on a set of 50 colorectal carcinoma cases [13]. The reproducibility was fair overall, and although cytokeratin staining increased tumor budding counts (partially by detection of single cells in glands disrupted by inflammatory cells), it did not increase the agreement between observers (Ueno methods hematoxylin and eosin $k=0.35 / 0.34$ and immunohistochemistry $k=$ $0.26 / 0.34)$ [13].

There has also been variation in the area in which tumor budding is assessed. Most studies have used the "hotspot method", as was used in the Ueno study [8, 9], whereby the pathologist decides on a single field that displays maximal budding to perform the count. This choice of field, however, may introduce variability. Other studies have examined multiple fields, ranging from assessment of the entire invasive front, to bud counts in 5-10 fields with use of the average count [10-12]. However, in the same multicenter study of tumor budding reproducibility, both the Ueno (hotspot) and the Wang (average of $5 \mathrm{hpf}$ ) methods had comparable interobserver variability $(k=0.35$ vs. 0.25$)$ [13].

The concept of whether "peritumoral" vs. "intratumoral" tumor budding, or both, should be assessed is still under discussion. The original studies on tumor budding, in pT1 colorectal carcinoma, utilized "peritumoral" budding, or assessment of budding at the invasive front of the carcinoma $[7,8]$. In the case of a malignant polyp, or pT1 colorectal carcinoma, this makes sense as the specimen is small and the invasive front is usually clear. However, the delineation of an invasive front in a biopsy or in a resection for pancreatic ductal adenocarcinoma, for example, would be difficult and poorly reproducible. Therefore, the idea of intratumoral budding was introduced in 2011, in the context of preoperative biopsies of colorectal carcinoma [14]. Intratumoral budding has been defined as clusters of less than five cells surrounded by tumor stroma within the tumor bulk. Giger et al. [14] showed intratumoral budding in the biopsy and peritumoral budding in the resection to be similarly reproducible ( $k=0.65$ vs. $k=0.68$ ), and to be significantly associated. Since then the concept of intratumoral budding has been applied in pancreatic ductal adenocarcinoma and esophageal squamous cell carcinoma.

The Ueno method, with some modifications, was the standardized method chosen at a recent consensus conference on the reporting of tumor budding in colorectal carcinoma [1]. The consensus definition of tumor budding was as follows based on use of hematoxylin and eosin staining: single cells or clusters of $<5$ cells, counted in the area of maximal budding ("hotspot method") at a $\times 200$ field (then normalized to a $0.785 \mathrm{~mm}^{2}$ field), with patients then classified as low grade ( $0-4$ buds), intermediate ( $5-9$ buds), and high-grade budding ( $\geq 10$ buds). To minimize variability in selecting the field of maximal budding, they suggest screening at least $10 \mathrm{hpf}$ at the invasive front. Although it was felt that intratumoral budding is likely also a useful prognostic factor, it was recommended that more research is needed before incorporation into routine practice. These were the recommendations for colorectal carcinoma, however, the optimal method for assessing tumor budding, and especially the optimal cutoff values, may vary in different sites of the gastrointestinal tract, and the different methods used in these malignancies will be discussed with the studies.

\section{Colorectal adenocarcinoma}

Tumor budding has been well established as a poor prognostic factor in colorectal carcinoma, with two well-studied clinical applications [15], and will be reviewed briefly here.

Early stage colorectal carcinoma, detected in polypectomy specimens as pT1 colorectal cancer, has the potential to be cured with polypectomy alone, although -9-24\% of these patients have lymph node metastases [9, 16-23]. High-grade tumor budding has been well established as one of the pathologic prognostic criteria that carry increased risk for lymph node metastases and, therefore, need for surgery post polypectomy (see Table 1) [7-9, 21, 23]. In a systematic review of 17 studies on the risk of lymph node metastases in pT1 colorectal carcinoma based on pathologic prognostic factors, tumor budding (investigated in seven studies) was found to be a significant risk factor for lymph node metastases, in addition to lymphatic invasion and submucosal invasive depth of $>1 \mathrm{~mm}$ [24]. Tumor budding carried a relative risk of 5.1 (3.6-7.3) for lymph node metastasis, and was an independent predictive factor in five of the six studies that performed a multivariate analysis. The Japanese Society for Cancer of 


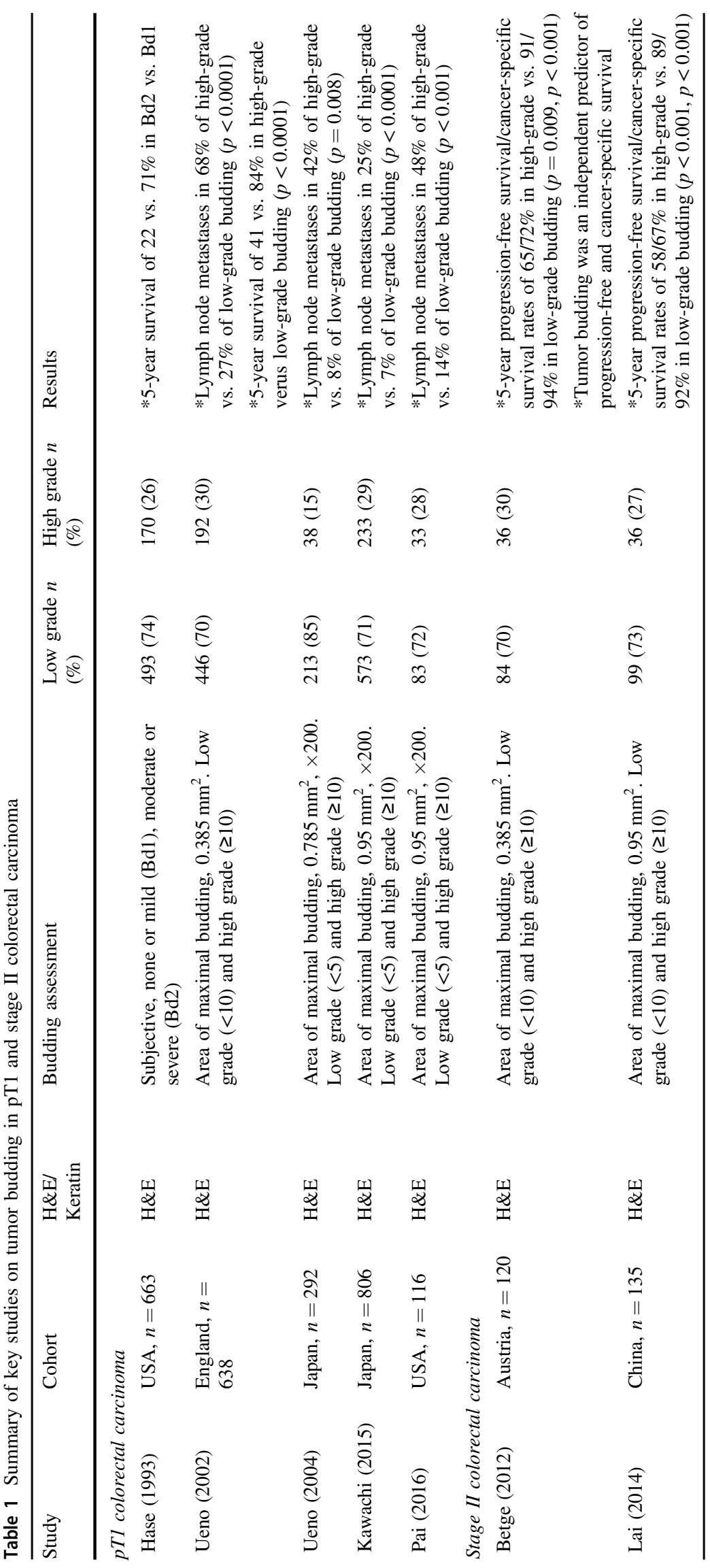


the Colon and Rectum has incorporated tumor budding into the guidelines for which pT1 carcinoma patients require subsequent surgery (along with vascular invasion, high grade, and submucosal depth of invasion $>1000 \mu \mathrm{m}$ ), using the consensus method for assessment of budding, and applying a cutoff of 5 or more (intermediate and high-grade categories) as high risk [25].

Stage II colorectal carcinoma encompasses a group of patients with T3-T4 tumors, and no lymph node metastases. Although some of these patients are cured with surgery, a subset of these patients have substantial risk of recurrence (5-year survival rates of 45-67\%, SEER database) [26]. Tumor budding has been well established as a poor prognostic factor in stage II colorectal carcinoma (see Table 1) $[27,28]$. A recent systematic review of 12 studies assessing tumor budding as a prognostic factor in stage II colorectal carcinoma showed high-grade budding to be a significant poor prognostic factor with an odds ratio of 6.25 for mortality (4.04-9.67, $p<0.00001)$ and to be an independent predictor of mortality (HR: 3.68, 2.16-6.26, $p<0.00001$ ) [29]. Tumor budding should be routinely reported in stage II CRC in order to aid in the decision for adjuvant therapy (utilizing a cutoff value of 10 , or high grade, per the consensus conference) along with other known predictive factors including mismatch repair status [1].

More recently, tumor budding has been studied in preoperative biopsies of colonic masses as a potential predictive factor for lymph node metastases, although the criteria for this application are unclear [14, 30, 31]. The methods for assessing tumor budding in these studies have been substantially different, using qualitative assessment of presence/absence of budding at low power vs., at the opposite end of the spectrum, quantitative assessment of budding using a cytokeratin stain at high power. All of these studies showed a significant association with lymph node metastases however, and hopefully future larger studies may standardize the approach to grading tumor budding in preoperative biopsies in order to establish budding as a clinical useful decision factor.

High-grade tumor budding has also been reported to predict non-responders to anti-EGFR therapies, along with K-Ras mutation status [32]. Of interest in that study, low-grade tumor budding was $100 \%$ predictive of response to EGFR therapy, with all low-grade budding having at least partial response, and no progressive or even stable disease [32].

\section{Esophageal squamous cell carcinoma and adenocarcinoma}

Multiple studies have now associated high-grade tumor budding with a poor prognosis for esophageal squamous cell carcinoma (see Table 2 for summary), and more recently tumor budding has been incorporated into a grading system for esophageal squamous cell carcinoma. The majority of the studies on esophageal squamous cell carcinoma have utilized a modified Ueno method, defining tumor budding to be single cells to clusters of $<5$, counted in the area of maximal budding at $\times 200$ and classified into low-grade/rare budding ( $<5$ buds) and high-grade/frequent budding ( $\geq 5$ buds).

The significance of tumor budding as a poor prognostic factor in cohorts of all patients undergoing esophagectomy for esophageal squamous cell carcinoma has been replicated in multiple studies. Roh et al. [33] first studied a cohort of 56 patients (39\% low-grade/61\% high-grade budding), and found the 3 -year survival rate to be significantly lower in high-grade budding (30.7 vs. $72.3 \%, p=0.04)$. Tumor budding was independent of differentiation, but was associated with larger tumor size. Another group replicated these findings in a larger cohort of 136 patients (40\% lowgrade $/ 60 \%$ high-grade budding) [34]. The 3- and 5-year survival rates for the high-grade group $(43,35 \%)$ were significantly higher than the low-grade group (92, 81\%, $p<0.001)$. Lymph node metastases, lymphovascular invasion, and higher $\mathrm{T}$ stage were all significantly associated with high-grade budding. By multivariate analysis, tumor budding (HR: 4.195, 1.77-9.941) and lymph node metastases (HR: 4.682) were found to be independent prognostic factors. More recently, a smaller cohort of 82 patients (44\% low-grade $/ 56 \%$ high-grade budding) showed significantly lower disease-free survival in the high-grade budding group $(p<0.0001)$ with a trend toward decreased overall survival [35]. These studies have speculated on, but not directly addressed, the use of high-grade tumor budding in the decision for adjuvant therapy, but future studies are required to investigate this potential application.

One small cohort study $(n=74)$ has shown tumor budding in the esophagectomy specimen to maintain its value as a significant prognostic factor even after neoadjuvant chemotherapy [36]. High-grade budding was correlated with a significantly lower 5-year survival (17 vs. 58\%, $p<0.001)$. This study also correlated tumor budding with neoadjuvant response, dividing patient based on clinical staging into complete response, partial response, and no response. Promisingly, high-grade budding was found to be significantly less common in responders to neoadjuvant therapy than non-responders $(p=0.001)$, and this raises the potential that tumor budding might be useful as a predictive marker of response to neoadjuvant therapy. However, as the tumor budding analysis was limited to the final resection specimen without assessment of the biopsy, this remains a question for a future study.

Tumor budding in pT1 esophageal squamous cell carcinoma has been investigated in one study, in an attempt to determine factors that might allow some T1 patients to be 


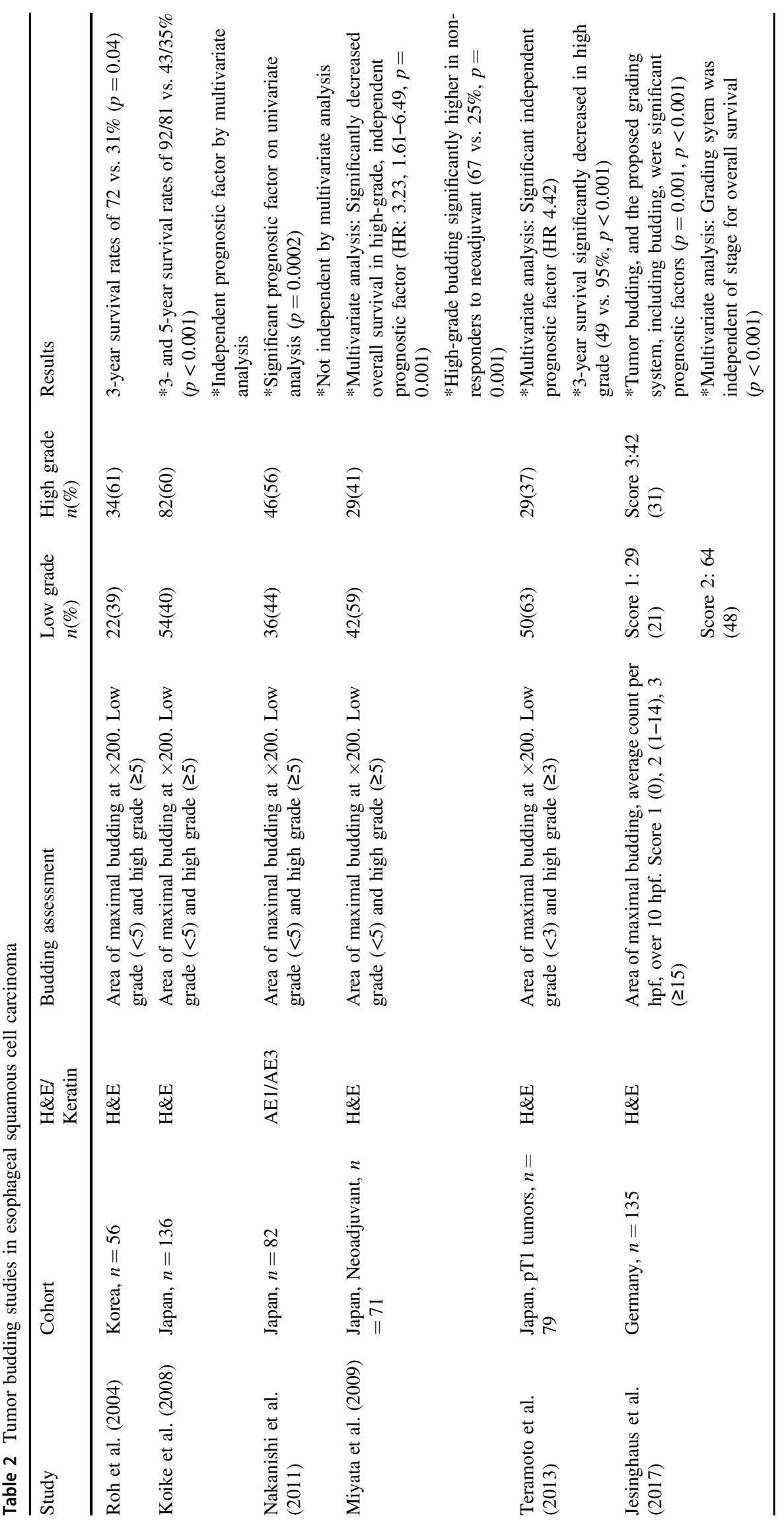




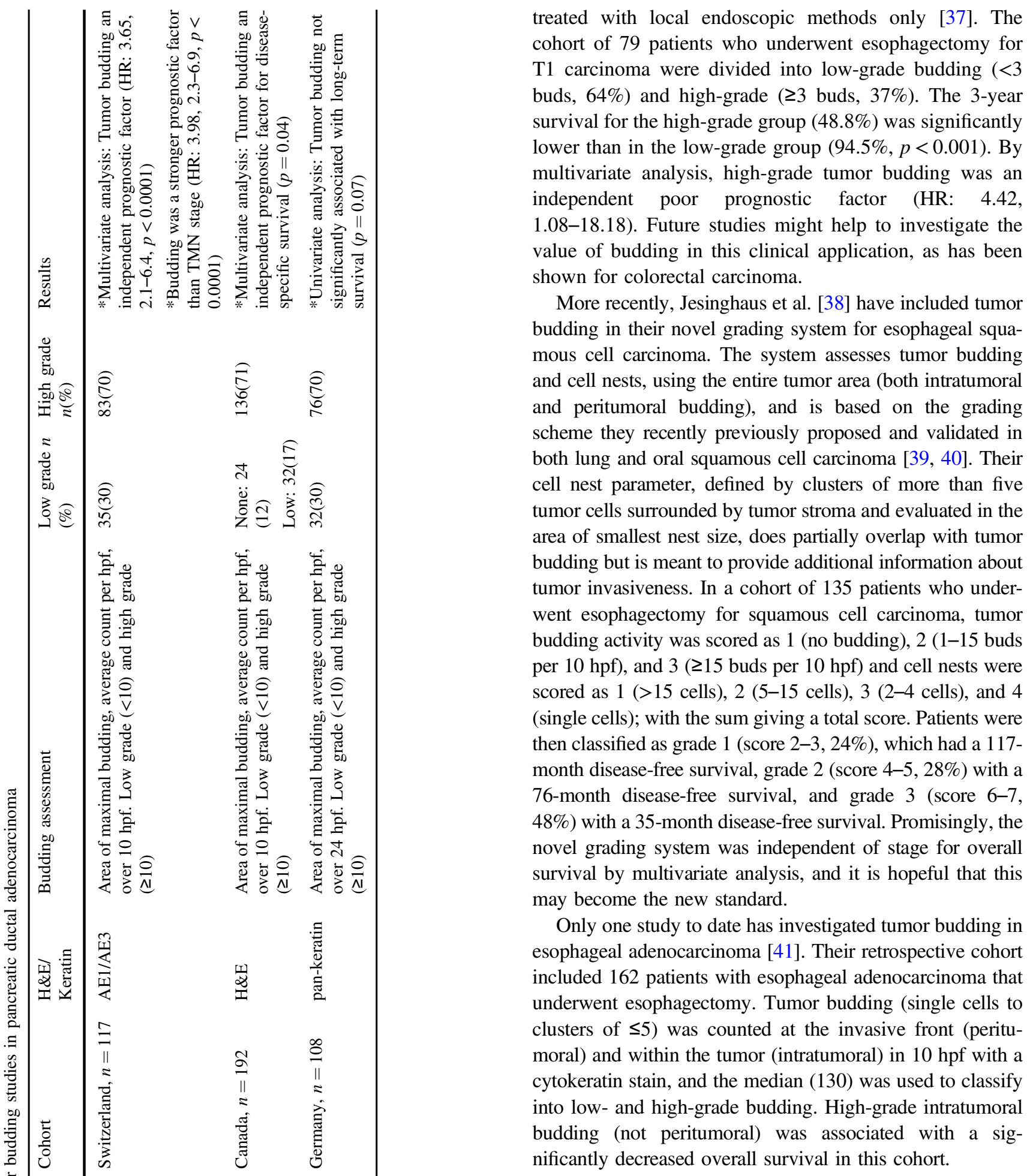

Gastric adenocarcinoma

Only in the last few years has tumor budding been investigated as a potential prognostic factor in gastric adenocarcinoma. Tumor budding in gastric adenocarcinoma is only assessed in the well-differentiated/ 
intestinal subtype, as the undifferentiated/diffuse type by definition displays tumor budding.

Early gastric adenocarcinomas (T1a) have the potential to be treated and cured with endoscopic mucosal resection or endoscopic submucosal dissection alone, with current criteria including size $<2 \mathrm{~cm}$ without ulceration, intestinal type, and absence of lymphovascular invasion; however, currently $\mathrm{T} 1 \mathrm{~b}$ gastric adenocarcinomas are all treated with subtotal or total gastrectomy without consideration for local therapy [42]. A study by Gulluoglu et al. [43] assessed the ability of tumor budding to predict lymph node metastases in $126 \mathrm{~T} 1$ gastric adenocarcinomas that had undergone gastrectomy. They used a different method for assessing budding than any other study reviewed here, looking for budding (single cells or clusters of $<5$ ) at the invasive front, and counting the patient as budding positive if any buds were seen at $\times 400$ ( $31 \%$ of 126 cases). They found that tumor budding positivity was significantly more common in lymph node positive vs. node negative patients with pT1b carcinomas (76 vs. $24 \%, p<0.001$ ), and suggest with further study it may be one criterion could stratify T1b patients into low-risk candidates to undergo local treatment.

The utility of tumor budding as a general poor prognostic factor in cohorts of all patients undergoing gastrectomy has been replicated in three studies, all using different methods of budding assessment. A cohort of 52 gastric intestinaltype adenocarcinomas was assessed using budding counts in ten $\times 200$ fields, with an average count of $\geq 1$ bud per field considered positive for high-grade budding (63\% of cases) [44]. High-grade tumor budding was significantly associated with lymph node metastasis (55 vs. $5 \%, p<0.001$ ). A larger cohort of 153 well-differentiated gastric adenocarcinomas was assessed using an average bud count over at least three $\times 400$ fields in the area of maximal budding using a cytokeratin stain, with 10 buds per hpf as the cutoff for high-grade budding as determined by their receiver operating characteristic (ROC) curve. High-grade tumor budding was significantly associated with decreased overall survival (HR: 1.61, 1.12-2.41, $p<0.01$ ). A third study with a cohort of 296 patients with any T stage gastric adenocarcinoma showed a decreased overall survival (HR: 2.260, 1.617-3.159) with high-grade budding, however, this study had a combined cohort of intestinal and diffuse-type adenocarcinoma so interpretation of their results is difficult due to the confounding factor of known poor prognosis for diffuse-type vs. intestinal-type adenocarcinoma [45].

\section{Ampullary carcinoma}

Ampullary carcinoma is a rare gastrointestinal carcinoma with a wide range in expected survival rates, and tumor budding has been reported to be an independent prognostic factor [46]. One study to date has looked at tumor budding as a prognostic indicator in ampullary adenocarcinomas, using a multi-institutional cohort of 244 patients with true primary ampullary adenocarcinomas (epicenter in the ampulla or major papilla, or involving the papilla-Vater mucosa) [46]. A modified Ueno method to assess budding was used, defining high-grade budding to be an average of $\geq 3$ buds per $\times 200$ field ( $80 \%$ of cases). Patients with highgrade budding had increased lymph node metastases (44 vs. $17 \%, p<0.001$ ), and decreased 3- and 5-year survival rates (41 and $24 \%$ vs. 93 and $68 \%$ for low-grade budding, $p<0.001)$.

\section{Pancreatic ductal adenocarcinoma}

Although pancreatic cancer represents only 3\% of malignancies overall, it has an extremely poor prognosis, and is the fourth leading cause of cancer death [47]. There is a need to identify patients that might respond to aggressive treatment. Tumor budding has been investigated in multiple studies now as a prognostic marker in pancreatic ductal adenocarcinoma (see Table 3 for summary). All studies on budding in pancreatic ductal adenocarcinoma have not distinguished between peritumoral budding and intratumoral budding due to the difficulty and poor reproducibility in determining the invasive front, and the methods for assessing tumor budding have differed.

Tumor budding in pancreatic ductal adenocarcinoma was first investigated by Karamitopoulou et al. [10] in a cohort of 117 patients, using cytokeratin staining to count tumor budding in $10 \mathrm{hpf}$, and selecting $\geq 10$ buds per hpf as the cutoff for high-grade budding by receiver operating characteristic curve analysis. High-grade budding had a dramatic impact on survival, with a 1-year survival rate of $28 \%$ for high-grade budding vs. $86 \%$ for low-grade budding. By multivariate analysis of overall survival, tumor budding was the most powerful prognostic factor (HR: 3.98, 2.3-6.9), greater than TMN stage (HR: 1.2). This was replicated in a cohort of 192 pancreatic ductal adenocarcinoma patients, counting tumor budding on hematoxylin and eosin staining in $10 \mathrm{hpf}$, and classified budding into absent (12\% of cases), low grade $(<10$ buds per $10 \mathrm{hpf}, 17 \%$ of cases), or high grade ( $>10$ buds per $10 \mathrm{hpf}, 71 \%$ of cases) [48]. High-grade budding was significantly associated with a poorer prognosis, with disease-specific survival rates of 1.24 year (high grade) vs. 1.42 year (low grade, $p=0.039$ ).

The biologic basis for tumor budding in pancreatic ductal adenocarcinoma, and its similarity to that seen in colorectal carcinoma, has also been studied. Kohler et al. [49] studied a cohort of 108 patients with pancreatic ductal adenocarcinoma of the pancreatic head who had not received 
neoadjuvant therapy, using the Karamitopolou method. High-grade tumor budding was seen in $70 \%$ of cases, with a trend toward decreased survival that did not reach significance $(p=0.07)$. Interestingly however, this group also looked at loss of e-cadherin expression as a biologic marker of epithelial-mesenchymal transition, and found e-cadherin expression to be significantly lower in areas of tumor budding ( 23 vs. $88 \%, p<0.0001)$. More recently, the expression of e-cadherin and microRNAs in a small subset of pancreatic ductal adenocarcinomas exhibiting tumor budding was analyzed, using non-budding pancreatic ductal adenocarcinomas as a control [50]. Pancreatic ductal adenocarcinomas exhibiting tumor budding had decreased expression of e-cadherin, increased expression of e-cadherin suppressive regulatory factors ZEB1 and ZEB2, and corresponding downregulation in miR-200 family miRNAs, which have been shown to function in growth suppression.

One group has looked at the association between FOXP3 + regulatory $\mathrm{T}$ cells and high-grade tumor budding in a group of 120 patients with pancreatic ductal adenocarcinoma, using the Karamitopolou method [51]. Increased regulatory $\mathrm{T}$ cell counts were significantly associated with high-grade tumor budding $(p=0.04)$, and worse prognosis by univariate analysis $(p=0.03)$.

While tumor budding has now been well established as a poor prognostic factor in pancreatic ductal adenocarcinoma, clinically relevant applications to the management of patients have not been established. Part of the difficulty lies in the differing methods of budding assessment used in the studies, and hopefully consensus can be achieved in pancreatic ductal adenocarcinoma as has been done for colorectal carcinoma. Studies on tumor budding in pancreatic ductal adenocarcinoma have speculated that tumor budding in pancreatic ductal adenocarcinoma may be useful in selecting the small number of patients that may benefit from more aggressive treatment, and uniform reporting of budding may help with future studies and to develop this potential important clinical application [49].

\section{Conclusions}

Tumor budding is emerging as an important histomorphologic marker of an aggressive tumor behavior in the majority of gastrointestinal carcinomas. We are now in the fortunate situation to have a standardized scoring system for colorectal carcinoma that will hopefully allow for comparative studies to demonstrate meaningful and standardized assessment methods and cutoff values for tumor budding in other gastrointestinal carcinomas. Only robust clinically annotated samples assessed for tumor budding will allow for exact substratification of patients, and as such it is of utmost importance to confirm and reproduce cutoff counts of this important histomorphologic parameter.

This review aims to serve as summary of the current state-of-tumor budding along the entire gastrointestinal tract. With respect to colorectal carcinoma, pathologists should now be routinely assessing tumor budding utilizing the consensus method in both pT1 and stage II colorectal carcinoma. In regard to esophageal squamous cell carcinoma, well-differentiated gastric adenocarcinoma, ampullary adenocarcinoma, and pancreatic ductal adenocarcinoma, multiple potential clinical applications for tumor budding have been studied and reviewed here. It will require awareness of these studies and of the utility of tumor budding beyond colorectal carcinoma to allow for wide spread implementation of this marker into routine surgical pathology reports in other areas of the gastrointestinal tract. However, it must be stressed that this is not "just" a pathology issue but rather will require close collaboration between pathologists, surgeons, and oncologists to further validate potential applications of this inexpensively assessed and powerful pathologic parameter.

\section{Compliance with ethical standards}

Conflict of interest The authors declare that they have no conflict of interest.

\section{References}

1. Lugli A, Kirsch R, Ajioka Y, et al. Recommendations for reporting tumor budding in colorectal cancer based on the International Tumor Budding Consensus Conference (ITBCC) 2016. Mod Pathol. 2017;30:1299-311.

2. Imai T. The growth of human carcinoma: a morphological analysis. Fukuoka Igaku Zasshi. 1954;45:72-102.

3. Brabletz T, Jung A, Reu S, et al. Variable $\beta$-catenin expression in colorectal cancers indicates tumor progression driven by the tumor environment. PNAS. 2001;98:10356-61.

4. Smedt L, Palmans S, Andel D, et al. Expression profiling of budding cells in colorectal cancer reveals an EMT-like phenotype and molecular subtype switching. $\mathrm{Br} \mathrm{J}$ Cancer. 2016;116:58-65.

5. Yamada N, Sugai T, Eizuka M, et al. Tumor budding at the invasive front of colorectal cancer may not be associated with the epithelial-mesenchymal transition. Hum Pathol. 2017;60:151-9.

6. Craene B, Berx G. Regulatory networks defining EMT during cancer initiation and progression. Nat Rev Cancer. 2013;13:97-110.

7. Hase K, Shatney C, Johnson D, et al. Prognostic value of tumor 'budding' in patients with colorectal cancer. Dis Colon Rectum. 1993;36:627-35.

8. Ueno H, Murphy J, Jass J, et al. Tumour 'budding' as an index to estimate the potential of aggressiveness in rectal cancer. Histopathology. 2002;40:127-32.

9. Ueno H, Mochizuki H, Hashiguchi Y, et al. Risk factors for an adverse outcome in early invasive colorectal carcinoma. Gastroenterology. 2004;127:385-94. 
10. Karamitopoulou E, Zlobec I, Born D, et al. Tumour budding is a strong and independent prognostic factor in pancreatic cancer. Eur J Cancer. 2013;49:1032-9.

11. Nakamura T, Mitomi H, Kikuchi S, et al. Evaluation of the usefulness of tumor budding on the prediction of metastasis to the lung and liver after curative excision of colorectal cancer. Hepatogastroenterology. 2005;52:1432-5.

12. Wang LM, Kevans D, Mulcahy $\mathrm{H}$, et al. Tumor budding is a strong and reproducible prognostic marker in T3NO colorectal cancer. Am J Surg Pathol. 2009;33:134-41.

13. Puppa G, Senore C, Sheahan K, et al. Diagnostic reproducibility of tumour budding in colorectal cancer: a multicentre, multinational study using virtual microscopy. Histopathology. 2012;61:562-75.

14. Giger O, Comtesse S, Lugli A, et al. Intra-tumoral budding in preoperative biopsy specimens predicts lymph node and distant metastasis in patients with colorectal cancer. Mod Pathol. 2012;25:1048-53.

15. Koelzer V, Zlobec I, Lugli A. Tumor budding in colorectal cancer —ready for diagnostic practice? Hum Pathol. 2016;47:4-19.

16. Nivatvongs S, Rojanasakul A, Reiman HM, et al. The risk of lymph node metastasis in colorectal polyps with invasive adenocarcinoma. Dis Colon Rectum. 1991;34:323-8.

17. Nascimbeni R, Burgart LJ, Nivatvongs S, et al. Risk of lymph node metastasis in T1 carcinoma of the colon and rectum. Dis Colon Rectum. 2002;45:200-6.

18. Toh E-W, Brown P, Morris E, et al. Area of submucosal invasion and width of invasion predicts lymph node metastasis in pT1 colorectal cancers. Dis Colon Rectum. 2015;58:393-400.

19. Kitajima K, Fujimori T, Fujii S, et al. Correlations between lymph node metastasis and depth of submucosal invasion in submucosal invasive colorectal carcinoma: a Japanese collaborative study. J Gastroenterol. 2004;39:534-43.

20. Wang H-S, Liang W-Y, Lin T-C, et al. Curative resection of T1 colorectal carcinoma: risk of lymph node metastasis and long-term prognosis. Dis Colon Rectum. 2005;48:1182-92.

21. Pai RK, Chen Y, Jakubowski MA, et al. Colorectal carcinomas with submucosal invasion (pT1): analysis of histopathological and molecular factors predicting lymph node metastasis. Mod Pathol. 2016;30:113-22.

22. Tsuruta O, Tsuji Y, Kawano H, et al. Indication for endoscopic resection of submucosal colorectal carcinoma: special reference to lymph node metastasis. Diagn Ther Endosc. 2000;6:101-9.

23. Kawachi H, Eishi $\mathrm{Y}$, Ueno $\mathrm{H}$, et al. A three-tier classification system based on the depth of submucosal invasion and budding/ sprouting can improve the treatment strategy for $\mathrm{T} 1$ colorectal cancer: a retrospective multicenter study. Mod Pathol. 2015;28:872-9.

24. Bosch S, Teerenstra S, Wilt J, et al. Predicting lymph node metastasis in pT1 colorectal cancer: a systematic review of risk factors providing rationale for therapy decisions. Endoscopy. 2013;45:827-41.

25. Watanabe T, Muro K, Ajioka Y, et al. Japanese Society for Cancer of the Colon and Rectum (JSCCR) guidelines 2016 for the treatment of colorectal cancer. Int J Clin Oncol. 2017; Epub ahead of print.

26. Edge SB, Byrd DR, Compton CC. Colon and rectum. In: Edge SB, Byrd DR, Compton CC, editors. AJCC cancer staging manual. 7th ed. New York, NY: Springer; 2010. p. 143-64.

27. Betge J, Kornprat $P$, Pollheimer M, et al. Tumor budding is an independent predictor of outcome in AJCC/UICC stage II colorectal cancer. Ann Surg Oncol. 2012;19:3706-12.

28. Lai YH, Wu LC, Li PS, et al. Tumour budding is a reproducible index for risk stratification of patients with stage II colon cancer. Colorectal Dis. 2014;16:259-64.
29. Petrelli F, Pezzica E, Cabiddu M, et al. Tumour budding and survival in stage II colorectal cancer: a systematic review and pooled analysis. J Gastrointest Cancer. 2015;46:212-8.

30. Rogers A, Gibbons D, Hanly A, et al. Prognostic significance of tumor budding in rectal cancer biopsies before neoadjuvant therapy. Mod Pathol. 2014;27:156-62.

31. Zlobec I, Hädrich $\mathrm{M}$, Dawson $\mathrm{H}$, et al. Intratumoural budding (ITB) in preoperative biopsies predicts the presence of lymph node and distant metastases in colon and rectal cancer patients. $\mathrm{Br}$ J Cancer. 2013;110:1008-13.

32. Zlobec I. Tumor budding predicts response to anti-EGFR therapies in metastatic colorectal cancer patients. World J Gastroenterol. 2010;16:4823-31.

33. Roh MS, Lee JI, Choi PJ. Tumor budding as a useful prognostic marker in esophageal squamous cell carcinoma. Dis Esophagus. 2004; 17:333-7.

34. Koike M, Kodera Y, Itoh Y, et al. Multivariate analysis of the pathologic features of esophageal squamous cell cancer: tumor budding is a significant independent prognostic factor. Ann Surg Oncol. 2008;15:1977-82.

35. Nakanishi Y, Ohara M, Doumen H, et al. Correlation between tumor budding and post-resection prognosis in patients with invasive squamous cell carcinoma of the thoracic esophagus. World J Surg. 2011;35:349-56.

36. Miyata H, Yoshioka A, Yamasaki M, et al. Tumor budding in tumor invasive front predicts prognosis and survival of patients with esophageal squamous cell carcinomas receiving neoadjuvant chemotherapy. Cancer. 2009;115:3324-34.

37. Teramoto $\mathrm{H}$, Koike $\mathrm{M}$, Tanaka $\mathrm{C}$, et al. Tumor budding as a useful prognostic marker in T1-stage squamous cell carcinoma of the esophagus. J Surg Oncol. 2013;108:42-46.

38. Jesinghaus M, Boxberg M, Konukiewitz B, et al. A novel grading system based on tumor budding and cell nest size is a strong predictor of patient outcome in esophageal squamous cell carcinoma. Am J Surg Pathol. 2017;41:1112-20.

39. Weichert W, Kossakowski C, Harms A, et al. Proposal of a prognostically relevant grading scheme for pulmonary squamous cell carcinoma. Eur Respir J. 2016;47:938-46.

40. Boxberg M, Jesinghaus M, Dorfner C, et al. Tumour budding activity and cell nest size determine patient outcome in oral squamous cell carcinoma: proposal for an adjusted grading system. Histopathology. 2017;70:1125-37.

41. Thies S, Guldener L, Slotta-Huspenina J, et al. Impact of peritumoral and intratumoral budding in esophageal adenocarcinomas. Hum Pathol. 2016;52:1-8.

42. Smyth E, Verheij M, Allum W, et al. Gastric cancer: ESMO clinical practice guidelines for diagnosis, treatment and follow-up. Ann Oncol. 2016;27:v38-v49.

43. Gulluoglu M, Yegen G, Ozluk Y, et al. Tumor budding is independently predictive for lymph node involvement in early gastric cancer. Int J Surg Pathol. 2015;23:349-58.

44. Olsen S, Linda J, Fields RC, et al. Tumor budding in intestinal type gastric adenocarcinoma is associated with nodal metastasis and recurrence. Hum Pathol. 2017;68:26-33.

45. Che K, Zhao Y, Qu X, et al. Prognostic significance of tumor budding and single cell invasion in gastric adenocarcinoma. Onco Targets Ther. 2017;10:1039-47.

46. Ohike N, Coban I, Kim GE, et al. Tumor budding as a strong prognostic indicator in invasive ampullary adenocarcinomas. Am J Surg Pathol. 2010;34:1417.

47. Siegel RL, Miller KD, Jemal A. Cancer statistics, 2016. CA Cancer J Clin. 2016;66:7-30.

48. O'Connor K, Li-Chang HH, Kalloger SE, et al. Tumor budding is an independent adverse prognostic factor in pancreatic ductal adenocarcinoma. Am J Surg Pathol. 2015;39:472. 
49. Kohler I, Bronsert P, Timme S, et al. Detailed analysis of epithelial-mesenchymal transition and tumor budding identifies predictors of long-term survival in pancreatic ductal adenocarcinoma. J Gastroenterol Hepatol. 2015;30:78-84.

50. Karamitopoulou E, Haemmig S, Baumgartner U, et al. MicroRNA dysregulation in the tumor microenvironment influences the phenotype of pancreatic cancer. Mod Pathol. 2017;30:1116-25.
51. Wartenberg M, Zlobec I, Perren A, et al. Accumulation of FOXP3+T-cells in the tumor microenvironment is associated with an epithelial-mesenchymal-transition-type tumor budding phenotype and is an independent prognostic factor in surgically resected pancreatic ductal adenocarcinoma. Oncotarget. 2015;6:4190-201. 